Sharif University of Technology
Scientia Iranica
SCIENTIA

\title{
Mixed convection in a lid-driven cavity with sinusoidal boundary temperature at the bottom wall in the presence of magnetic field
}

\author{
S. Sivasankaran ${ }^{\mathrm{a}, *}$, S.S. Ananthan ${ }^{\mathrm{b}}$ and A.K. Abdul Hakeem ${ }^{\mathrm{c}}$ \\ a. Institute of Mathematical Sciences, University of Malaya, Kuala Lumpur 50603, Malaysia. \\ b. Department of Mathematics, Erode Sengunthar Engineering College, Thudupathi, 638 05\%, Tamil Nadu, India. \\ c. Department of Mathematics, Sri Ramakrishna Mission Vidhyalaya College of Arts and Science, Coimbatore 641020, India.
}

Received 15 October 2014; received in revised form 24 March 2015; accepted 26 October 2015

\section{KEYWORDS}

Mixed convection;

Magnetic field;

Non-uniform heating;

Lid-driven cavity.

\begin{abstract}
Mixed convection heat transfer in a lid-driven square cavity with sinusoidal boundary temperature at the bottom wall in the presence of magnetic field is investigated, numerically. The top wall is preserved at a lower temperature. The left and right sidewalls of the cavity are thermally insulated. Finite volume method is used to solve the mass, momentum, and energy equations. The heat transfer rate is examined by varying the dimensionless parameters of Richardson number and Hartmann number. The fluid flow and the heat transfer rate are strongly affected inside the cavity by the presence of magnetic field.
\end{abstract}

(C) 2016 Sharif University of Technology. All rights reserved.

\section{Introduction}

Mixed convection is a combination of forced and free convections where a flow is determined simultaneously by both outer forcing system and inner volumetric forces by the non-uniform density distribution of a fluid medium in a gravity field. Mixed convection flow and heat transfer problems in lid-driven cavities have been receiving a considerable attention in literature. This attention stems from their importance in vast technological engineering and natural applications such as solar ponds, dynamics of lakes, thermal-hydraulics of nuclear reactors, food processing, cooling of electronic devices, and float glass production. Prasad and Koseff [1] studied the mixed convection heat transfer process in a lid-driven cavity. They concluded that heat transfer within the cavity is independent of the Richardson numbers from 0.1 to 1000 . Khanafer et al. [2] numerically examined mixed convection heat

\footnotetext{
*. Corresponding author.

E-mail address: sd.siva@yahoo.com (S. Sivasankaran)
}

transfer in a lid-driven cavity. They obtained that the Reynolds number and Grashof number would either enhance or retard the energy transport process and drag force behavior depending on the conduct of the velocity cycle. Waheed [3] studied the mixed convective heat transfer in a rectangular enclosure driven by a continuously moving horizontal plate. It is found that the fluid flow and temperature distributions within the enclosure and heat flux on the heated wall are enhanced by the increase in the Richardson number. Sivakumar et al. [4] numerically studied the mixed convection in a lid-driven cavity with different lengths and locations of the heater. They observed that the heat transfer rate increases when the location of heater is at the middle or top on the left wall of the cavity. Abedini et al. [5] numerically studied the mixed convection in the annulus between eccentric rotating cylinders. They found that the heat transfer and fluid flow were affected by the orientation and eccentricity of the inner cylinder. Magneto-hydrodynamics (MHD) is the study of the dynamics of electrically conducting fluid in the presence of electromagnetic field. The effects of 
magnetic field on convective flow and heat transfer are essential in many areas of industry. Therefore, consideration of MHD is very importance in the study of convection flow. Rudraiah et al. [6] numerically investigated the effect of the magnetic field on natural convection in a rectangular enclosure. They found that the rate of heat transfer decreased in the presence of magnetic field. Al-Najem et al. [7] numerically studied the laminar natural convection in a tilted enclosure with transverse magnetic field. They found that the heat transfer mechanisms and the flow characteristics inside the tilted enclosures depended strongly upon both the strength of the magnetic field and the inclination angle. Significant suppression of the convective current can be obtained by applying strong magnetic field and the magnetic field has negligible effect on the heat transfer mechanism for small inclination angles. Chamkha [8] made an analysis for mixed convection in a square cavity in the presence of the magnetic field and an internal heat generation or absorption. It has been shown that the flow behavior and the heat transfer characteristics inside the cavity are strongly affected by the presence of magnetic field. Tagawa et al. [9] numerically discussed the Rayleigh-Benard convection of water in the presence of magnetic field. They observed that the average Nusselt number decreased by increase in the strength of the magnetic field when the enclosure was placed above the coil center. Sarris et al. [10] carried out a numerical study on unsteady natural convection of an electrically conducting fluid in a laterally and volumetrically heated square cavity under the influence of magnetic field. It was found that the flow oscillations were reduced or vanished by the increase in Hartmann numbers due to the damping effect of magnetic field. Mittal et al. [11] analyzed buoyancy-driven convection of liquid metals subjected to transverse magnetic field. Hossain et al. [12] carried out an investigation into the effect of surface tension on natural convection flow of an electrically conducting fluid in a rectangular enclosure under an externally imposed magnetic field with internal heat generation. They found that the change of direction of the external magnetic field from horizontal to vertical leads to decrease in the flow rates in both primary and secondary cells in the cavity.

$\mathrm{Xu}$ et al. [13] experimentally investigated the natural convection of molten gallium in the presence of magnetic field. They concluded that natural convection was suppressed in the presence of imposed magnetic field and the magnetic damping effect was increased by increasing the applied magnetic field strength. Jalil and Al-Tae'y [14] numerically discussed the natural convection of molten sodium in a square enclosure. It is found that the values of the Nusselt numbers depend on the magnetic field distribution and decrease when the Hartmann number is increased.
Sivasankaran and Ho [15] numerically analyzed the effects of temperature-dependent properties of water near its maximum density in the presence of uniform magnetic field on fluid flow and heat transfer. They concluded that an increase of Hartmann number results in a decrease in heat transfer rate. Sivasankaran et al. [16] numerically studied the magneto-convection of cold water in an open cavity with variable fluid properties. It is observed that the convection heat transfer is enhanced by thermo capillary force when buoyancy force is weakened. Sivasankaran and Bhuvaneswari [17] numerically studied the effect of thermally active zones and direction of the external magnetic field on hydro-magnetic convection in an enclosure. They concluded that the average Nusselt number decreases with an increase of the Hartmann number and it increases with increase of the Grashof number and aspect ratio. Sankar et al. [18] numerically investigated the effect of magnetic field on the buoyancy and thermo capillary driven convection of an electrically conducting fluid in an annular enclosure. Kefayati [19] numerically analyzed the effect of magnetic field on natural convection flow in a nanofluid filled inclined square cavity. He showed that the heat transfer is decreased by the increment of Hartmann number for various Rayleigh numbers and inclined angles. Sheikholeslami and Ganji [20] numerically examined Magneto-hydrodynamic flow in a permeable channel filled with nanofluid. It is found that velocity boundary layer thickness is decreased when Reynolds number and nanoparticle volume fraction are increased.

Mixed convection problems in enclosures with sinusoidal boundary conditions either on one side wall or on both side walls have been examined by several researchers. The features of non-uniform temperature distribution are observed in many areas such as solar energy collection, indoor thermal environments, and nuclear reactor systems. Deng and Chang [21] numerically investigated the natural convection in an airfilled rectangular enclosure with two spatially varying sinusoidal temperature distributions on the vertical left and right side walls. It is observed that the variation of amplitude or phase of the sinusoidal temperature distribution on one sidewall mainly affects the heat transfer on its own surface and it has little effect on the sidewall. Sivasankaran et al. [22] numerically studied the mixed convection in a lid-driven cavity with non-uniform heating on both sidewalls. They obtained that the average Nusselt number increased while increasing the amplitude ratio. Bhuvaneswari et al. [23] numerically analyzed magneto-convection in an enclosure with non-uniform heating on both side walls. They found that the heat transfer rate was increased on increasing the amplitude ratio. Sivasankaran et al. [24] numerically studied the mixed convection in a square cavity with sinusoidal temperature on vertical 
walls in the presence of magnetic field. They concluded that increasing the Hartmann number results in the decrease of the total heat transfer rate. Sivasankaran and Pan [25] found from the study on mixed convection in a porous lid-driven cavity with non-uniform heating on both side walls that the average Nusselt number increased on increasing the amplitude ratio. Sivakumar and Sivasankaran [26] carried out a numerical study on effect of non-uniform heating on mixed convection in an inclined lid-driven cavity. They revealed that the average Nusselt number was extensively increased on increasing inclination angle in the buoyancy-dominated mode. Sivasankaran and Pan [27] numerically discussed the natural convection of nanofluids in a cavity with sinusoidal temperature distributions on side walls. They concluded that the heat transfer rate was increased while increasing amplitude ratio and volume fraction of nanoparticles.

In all the above-mentioned previous studies, the convection flow and heat transfer in cavities with various thermal boundary conditions (uniform or nonuniform) has been numerically investigated in detail. To the best of the author's knowledge, mixed convection in a square cavity with sinusoidal boundary at the bottom wall in the presence of magnetic field has received less attention in literature. Therefore, the main objective of the present study is to investigate the effect of the moving wall's direction and sinusoidal thermal boundary condition at the bottom wall on mixed convection in a square lid-driven cavity in the presence of magnetic field.

\section{Mathematical modelling}

The physical configuration model is a two-dimensional square cavity of size $L$ filled with electrically conducting fluid as shown in the Figure 1. The length and

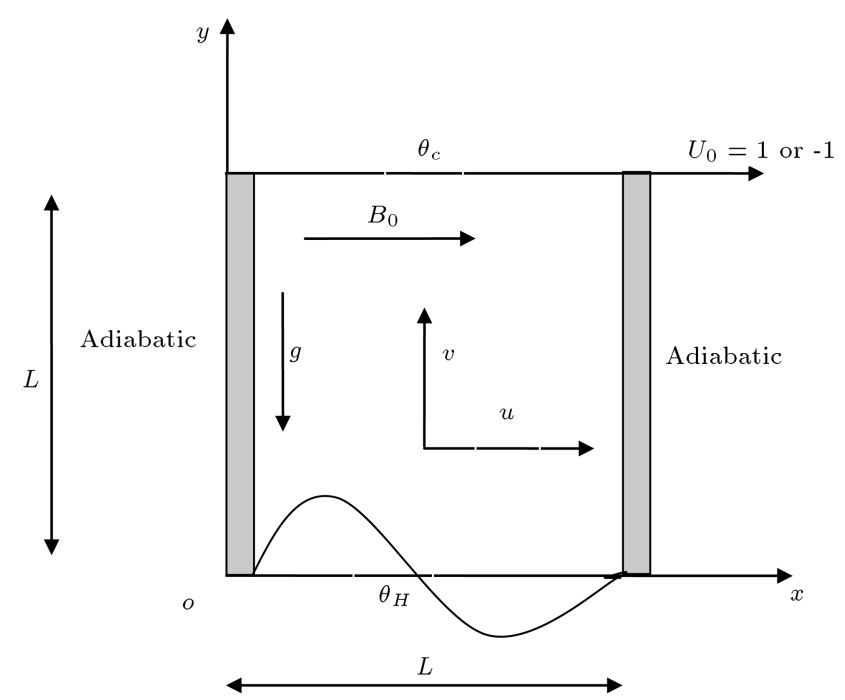

Figure 1. Schematic diagram. height of the cavity are taken to represent the axes $x$ and $y$, respectively. The velocity components in the directions of $x$ and $y$ are $u$ and $v$, respectively. The top wall of the cavity is moving in two directions (both right and left) at a constant speed $U_{0}$ in its own plane. The bottom wall is maintained at a varying sinusoidal temperature, $\theta_{H}$, (hot wall) and the top wall is kept at a temperature, $\theta_{C}$, (cold wall) with $\theta_{H}>\theta_{C}$. The left and right sidewalls of the cavity are insulated. A uniform magnetic field with a constant magnitude $B_{0}$ is applied in the horizontal direction. The gravitational acceleration acts downward. The density varies linearly with temperature as $\rho=\rho_{0}\left[1-\beta\left(\theta-\theta_{0}\right)\right]$, where $\beta$ being the coefficient of thermal expansion and subscript 0 denoting the reference state. The Boussinesq approximation is valid. The following assumptions are considered for the present study. Flow in the cavity is two-dimensional, laminar, and incompressible. The fluid properties are constant. The radiation, viscous dissipation, induced electric current, and Joule heating are neglected.

As per the above-mentioned assumptions, geometrical and physical conditions, the motion of twodimensional incompressible fluid is governed by the following equations:

$$
\begin{aligned}
& \frac{\partial u}{\partial x}+\frac{\partial v}{\partial y}=0 \\
& \frac{\partial u}{\partial t}+u \frac{\partial u}{\partial x}+v \frac{\partial u}{\partial y}=-\frac{1}{\rho} \frac{\partial p}{\partial x}+\nu\left(\frac{\partial^{2} u}{\partial x^{2}}+\frac{\partial^{2} u}{\partial y^{2}}\right) \\
& \frac{\partial v}{\partial t}+u \frac{\partial v}{\partial x}+v \frac{\partial v}{\partial y}=-\frac{1}{\rho} \frac{\partial p}{\partial y}+\nu\left(\frac{\partial^{2} v}{\partial x^{2}}+\frac{\partial^{2} v}{\partial y^{2}}\right) \\
& +g \beta\left(\theta-\theta_{C}\right)-\frac{\sigma_{e} B_{0}^{2} v}{\rho} \\
& \frac{\partial \theta}{\partial t}+u \frac{\partial \theta}{\partial x}+v \frac{\partial \theta}{\partial y}=\frac{k}{\rho c_{p}}\left(\frac{\partial^{2} \theta}{\partial x^{2}}+\frac{\partial^{2} \theta}{\partial y^{2}}\right)
\end{aligned}
$$

where $\theta$ is fluid temperature, $p$ is pressure, $k$ is thermal conductivity, $g$ is gravitational acceleration, $c_{p}$ is specific heat, and $t$ is time.

The dimensional initial and boundary conditions for the problem are written as follows:

For $t=0$ :

$$
u=v=0, \quad \theta=0, \quad 0 \leq x \leq y \leq L .
$$

For $t>0$ :

$$
\begin{aligned}
& u=v=0, \quad \frac{\partial \theta}{\partial x}=0, \quad x=0, \\
& u=v=0, \quad \frac{\partial \theta}{\partial x}=0, \quad x=L,
\end{aligned}
$$




$$
\begin{aligned}
& u=v=0, \quad \theta=\theta_{H}=\sin \left(\frac{2 \pi x}{L}\right), \quad y=0, \\
& u=U_{0} \text { or }-U_{0}, \quad v=0, \quad \theta=\theta_{C}=0, \quad y=L .
\end{aligned}
$$

To write the dimensional equations (Eqs. (1)-(4)) in a proper non-dimensional form, the following dimensionless variables are used.

$$
\begin{array}{lll}
X=\frac{x}{L}, & Y=\frac{y}{L}, & U=\frac{u}{U_{0}}, \\
V=\frac{v}{U_{0}}, & T=\frac{\theta-\theta_{C}}{\theta_{H}-\theta_{C}}, & \tau=\frac{t U_{0}}{L}, \\
\zeta=\omega L / U_{0}, & \Psi=\psi / L U_{0} . &
\end{array}
$$

After non-dimensionalization, the above governing equations can be rewritten in the vorticity and stream function as follows:

$$
\begin{aligned}
& \frac{\partial \zeta}{\partial \tau}+U \frac{\partial \zeta}{\partial X}+V \frac{\partial \zeta}{\partial Y}=\frac{1}{\operatorname{Re}}\left(\frac{\partial^{2} \zeta}{\partial X^{2}}+\frac{\partial^{2} \zeta}{\partial Y^{2}}\right) \\
&+\frac{\operatorname{Gr}}{\operatorname{Re}^{2}} \frac{\partial T}{\partial X}-\frac{\mathrm{Ha}^{2}}{\operatorname{Re}} \frac{\partial V}{\partial X} \\
& \nabla^{2} \Psi=-\zeta \\
& \frac{\partial T}{\partial \tau}+U \frac{\partial T}{\partial X}+V \frac{\partial T}{\partial Y}=\frac{1}{\operatorname{Pr} \operatorname{Re}}\left(\frac{\partial^{2} T}{\partial X^{2}}+\frac{\partial^{2} T}{\partial Y^{2}}\right),
\end{aligned}
$$

where:

$$
U=\frac{\partial \Psi}{\partial Y}, \quad V=-\frac{\partial \Psi}{\partial X}, \quad \text { and } \quad \zeta=\frac{\partial V}{\partial X}-\frac{\partial U}{\partial Y}
$$

The non-dimensional parameters in the above equations are defined as: $\mathrm{Gr}=g \beta\left(T_{H}-T_{C}\right) L^{3} / \nu^{2}$ is the Grashof number, $\mathrm{Ha}=B_{0} L \sqrt{\sigma_{e} / \mu}$ is the Hartmann number, $\operatorname{Pr}=\nu / \alpha$ is the Prandtl number, $\operatorname{Re}=U_{0} L / \nu$ is the Reynolds number. The non-dimensional initial and boundary conditions of the considered problem are written as:

$$
\begin{aligned}
& \tau=0 \text { : } \\
& U=0, \quad V=0, \quad T=0, \\
& 0 \leq X \leq 1, \quad 0 \leq Y \leq 1 . \\
& \tau>0 \text { : } \\
& \begin{array}{lll}
U=0, & V=0, \quad \frac{\partial T}{\partial X}=0, & X=0, \\
U=0, & V=0, \quad \frac{\partial T}{\partial X}=0, & X=1, \\
U=0, & V=0, \quad T=\sin (2 \pi X), & Y=0, \\
U=1 \text { or }-1, \quad V=0, \quad T=0, \quad Y=1 .
\end{array}
\end{aligned}
$$

The heat transfer rate across the enclosure is a very essential parameter in the thermal engineering applications. It is calculated by the Nusselt number. The local Nusselt number along the bottom (hot) wall is defined by:

$$
\mathrm{Nu}=\left(\frac{\partial T}{\partial Y}\right)_{Y=0}
$$

The total heat transfer rate across the enclosure is calculated by:

$$
\overline{\mathrm{Nu}}=\int \mathrm{Nu} d X
$$

\section{Numerical method}

The discretization of non-dimensional equations (Eqs. (7)-(10)) is performed by applying the finitevolume method. The upwind scheme and central difference scheme are implemented for the convection and diffusion terms, respectively. A uniform grid is taken in both $X$ and $Y$ directions to compute the numerical solutions of the considered problem. The grid points are varied from $41 \times 41$ to $161 \times 161$ to find the grid-independency solutions for $\mathrm{Ri}=1, \operatorname{Pr}=0.054$, and $\mathrm{Ha}=0$. The grid independence test showed that a grid of $81 \times 81$ size is enough to find the desired accuracy of results. The velocity components $U$ and $V$ at every grid point are evaluated using the central difference approximations. The above process is repeated until the following convergence criterion for temperature, vorticity, and stream function is satisfied:

$$
\left|\frac{\Phi_{i j}^{n+1}-\Phi_{i j}^{n}}{\Phi_{i j}^{n+1}}\right|<10^{-5}
$$

where $n$ is any time level and $\Phi$ represents the field variables $(T, \zeta$ or $\Psi)$. The trapezoidal rule is used to compute the average Nusselt number along the hot wall.

\section{Results and discussion}

In this paper, mixed convection in a square cavity with sinusoidal temperature distribution at the bottom wall in the presence of uniform external magnetic field is numerically studied. It is clear that the non-dimensional parameters of interest are Richardson number (Ri), Hartmann number $(\mathrm{Ha})$, and the direction of lid $(U=1$ or -1$)$. Throughout this study, the Prandtl number, $\operatorname{Pr}=0.054$, and Grashof number, $\mathrm{Gr}=10^{4}$, are fixed. The mode of convection is determined by the Richardson number, $\mathrm{Ri}=\mathrm{Gr} / \mathrm{Re}^{2}$, with $\mathrm{Ri}<1$ (forced convection), $\mathrm{Ri}=1$ (mixed convection), and $\mathrm{Ri}>1$ (natural convection), respectively. Calculations 


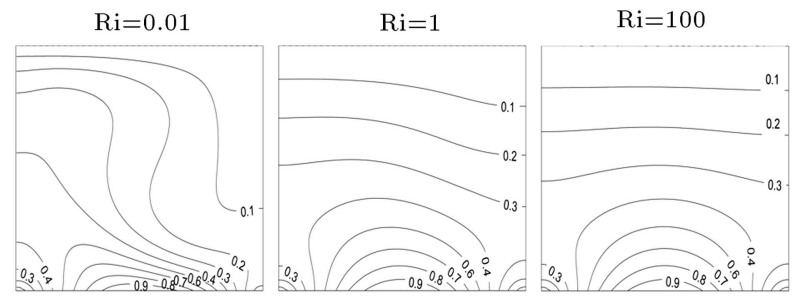

(a) $\mathrm{Ha}=0$

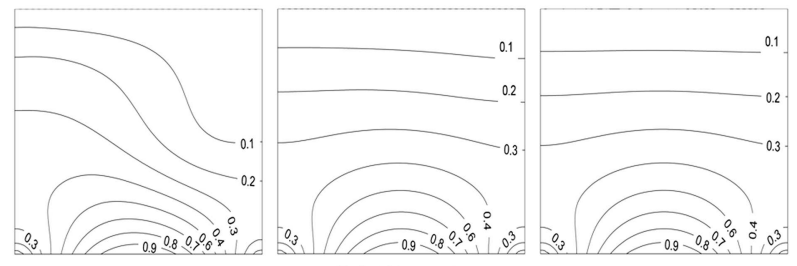

(b) $\mathrm{Ha}=20$

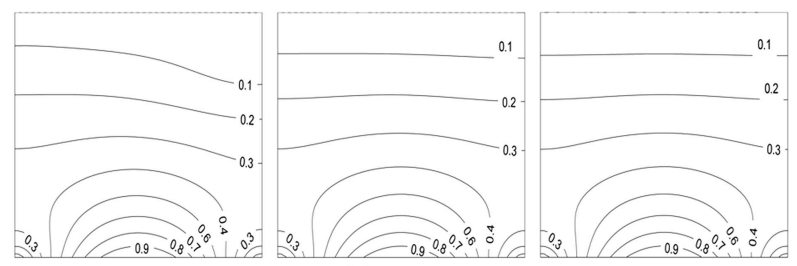

(c) $\mathrm{Ha}=50$

Figure 2. Isotherms for different Richardson numbers and Hartmann numbers with $U=1$.

through the cavity are made for a range of the Richardson numbers from 0.01 to 100 and the Hartmann numbers from 0 to 50 . The lid-driven cavity is analyzed according to the left and right directions of moving wall as shown in Figure 1.

\subsection{Effects of Richardson number and Hartmann number}

In this study, top wall is moving from left to right and right to left with velocity $U=1$ and $U=-1$. In Figures 2 and 3, the isotherms for various values of Richardson numbers and the Hartmann numbers are illustrated. When $\mathrm{Ri}=0.01$, thermal boundary layer is formed along the bottom wall of the cavity. It is observed that the heat distribution is along the vertical direction and is better near the left sidewall than the right sidewall in the absence of magnetic field and weak magnetic field, $\mathrm{Ha}=20$, for the top wall is moving from left to right direction $(U=1)$ in Figure 2. But, the heat distribution is along the vertical direction and is better near the right sidewall than the left sidewall in the absence of magnetic field and weak magnetic field for $\mathrm{Ha}=20$ when the top wall is moving from right to left direction $(U=-1)$ in Figure 3 . This reveals that the heat transfer is dominated by convection mode. Increasing the Hartmann number to $\mathrm{Ha}=50$, there is no considerable change in the temperature distribution for both moving directions of top wall. The temperature distribution seems to turn into conduction mode in the upper half of the

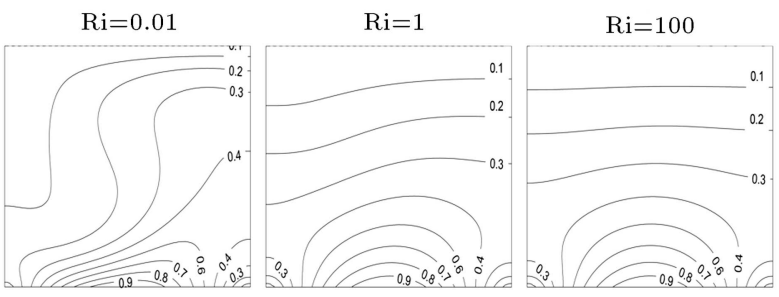

(a) $\mathrm{Ha}=0$

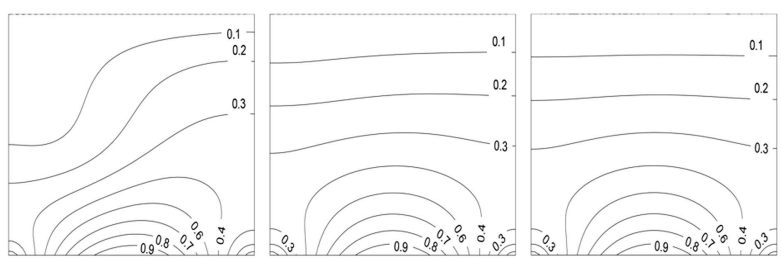

(b) $\mathrm{Ha}=20$

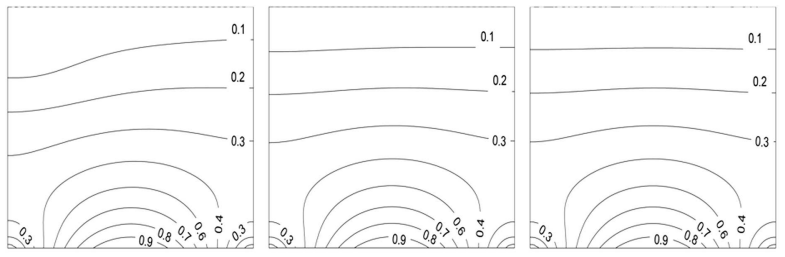

(c) $\mathrm{Ha}=50$

Figure 3. Isotherms for different Richardson numbers and Hartmann numbers with $U=-1$.

cavity. In the mixed convection region, the thickness of the thermal boundary layer along the thermally active bottom wall is decreased. The vertical temperature distribution inside the cavity disappears. The same behaviour of isotherms is found for all the values of Hartmann numbers from 0 to 50. Further, increasing the Richardson number to $\mathrm{Ri}=100$, there is no significant change in the temperature distribution, even when the Hartmann number is varied from 0 to 50 . It is clear that the heat distribution decreases as the magnetic field is increased. Temperature distribution is affected in the mixed and natural convection regions. As a result, the heat transfer is mostly in conduction mode in the cavity. Also, the convection is suppressed in the presence of strong magnetic field.

The streamlines for different values of Richardson number and Hartmann number are displayed in Figures 4 and 5 . When $\mathrm{Ri}=0.01$, a single major circulating cell in the clockwise and anticlockwise directions appears in the entire cavity when magnetic field is not present as per the top wall velocities $U=1$ and -1 . Further, the circulation of flow field is gradually restrained by varying the Hartmann numbers to 20 and 50, except in the region near the moving top wall of the cavity. It is observed that the applied magnetic field plays the dominant role to shrink and down the movement of the fluid in the cavity. In the mixed convection region, the major cell is diagonally elongated in the absence of magnetic field. This happens due to the shear force caused by movement of 

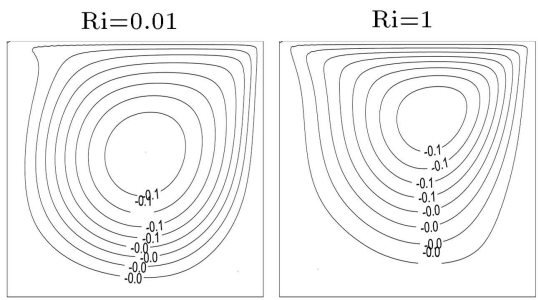

(a) $\mathrm{Ha}=0$
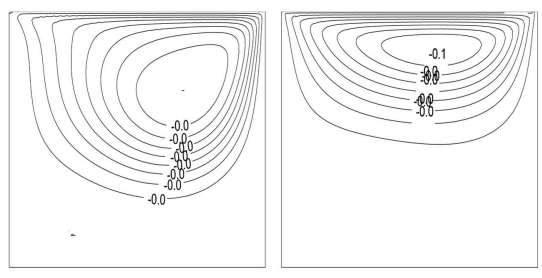

(b) $\mathrm{Ha}=20$
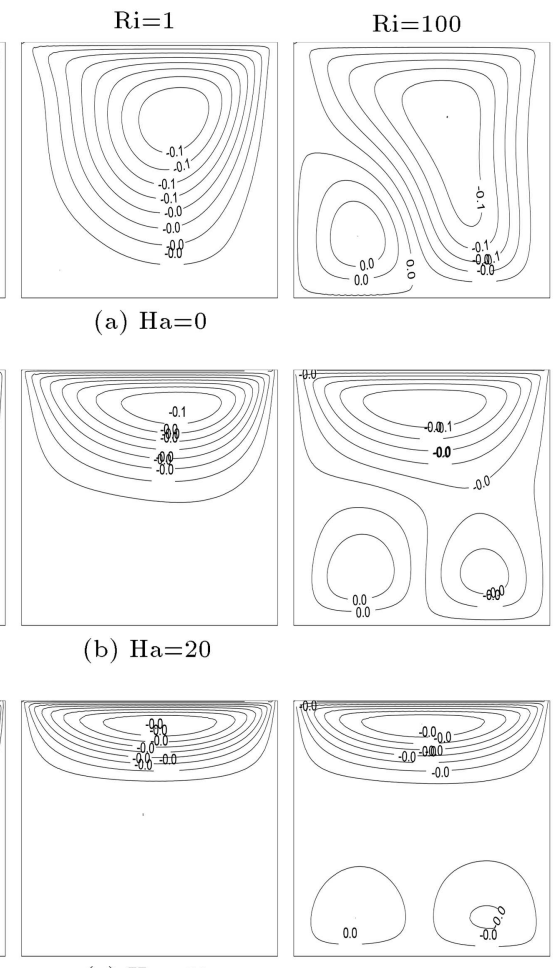

(c) $\mathrm{Ha}=50$

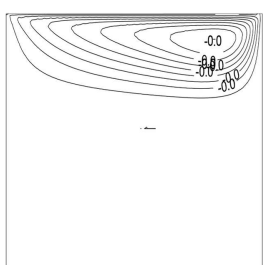

Figure 4. Streamlines for different Richardson numbers and Hartmann numbers with $U=1$.

the top wall. The cell shrinks by increase in the values of the Hartmann numbers to $\mathrm{Ha}=20$ and $\mathrm{Ha}=50$ in the forced convection region. Further, the core region of the cell is moving towards either right-top corner or left-top corner of the cavity, according to the lid moving direction $U=1$ and $U=-1$ when increasing the Hartmann number for $\mathrm{Ri}=0.01$. It reveals that the circulation of cell is dominated by the shear force due to the moving wall of the cavity. The flow is almost stagnant at the bottom of the cavity in the forced and mixed convection regions. When $\mathrm{Ri}=100$, twocellular flow structure is found for $\mathrm{Ha}=0$. The right upper corner cell is vertically elongated and again it forms two-cellular flow structure in the weak magnetic field at $\mathrm{Ha}=20$. When $\mathrm{Ha}=50$, size of the right bottom corner cell is decreased and left-bottom corner cell is slightly increased for the top wall velocity $U=1$. When the top wall is moving with velocity $U=-1$ in the free convection region, two-cellular flow structure is found for $\mathrm{Ha}=0$. Although, the size of the leftbottom corner cell is decreased and that of the rightbottom corner cell is slightly increased for $\mathrm{Ha}=50$ at $U=-1$. However, significant change is observed in the flow field when changing the Hartmann number in the free convection regime. It is clear that the flow field is strongly affected in the free convection region by the presence of magnetic field.

The local Nusselt number along the thermally active wall for different values of Richardson numbers and
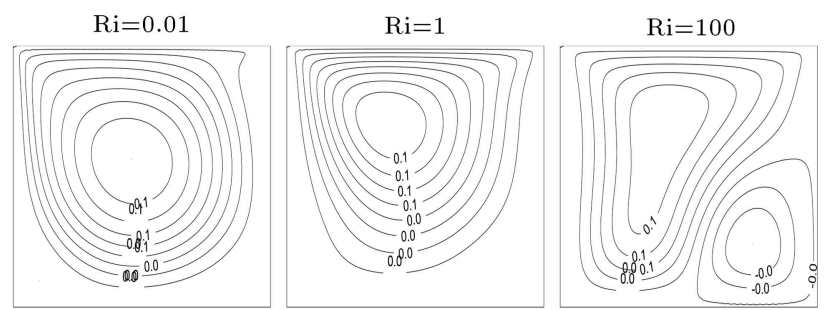

(a) $\mathrm{Ha}=0$
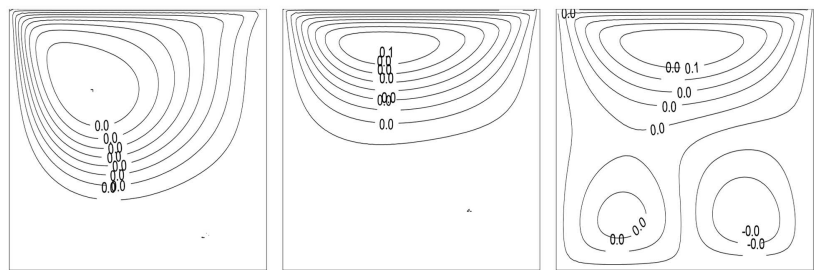

(b) $\mathrm{Ha}=20$
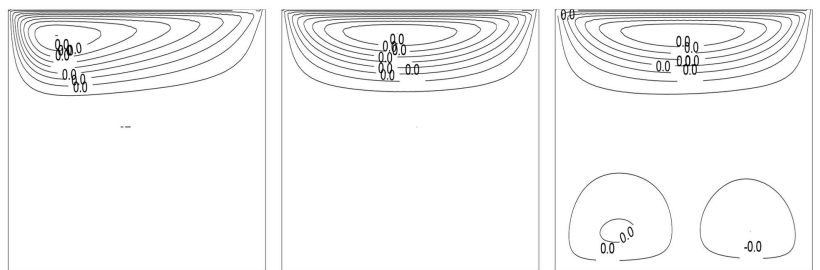

(c) $\mathrm{Ha}=50$

Figure 5. Streamlines for different Richardson numbers and Hartmann numbers with $U=-1$.

Hartmann numbers is presented in Figure 6(a)-(f). The curves drawn for the Nusselt numbers against $X$ axis are approximately of sinusoidal shape like the thermal boundary. In the forced convection region, the heat transfer at the bottom wall gets remarkable variations if the Hartmann number is increased (see Figure 6(a) and (b)). The local Nusselt number decreases on increasing the values of Hartmann number. It is observed that the Hartmann numbers have great influence on the heat transfer at the bottom wall. But, in the case of mixed and free convection modes, the heat transfer rate gets no remarkable variations while the Hartmann number is increased. These have been depicted in Figure 6(c) to (f). Further scrutinizing Figure 6(a)-(f), the local heat transfer rate is enhanced at the left, right, and middle regions of the bottom wall for all values of the Hartmann number.

The average Nusselt number is plotted in Figure $7(\mathrm{a})$ and (b) for different values of Richardson number and Hartmann number with top wall velocity $U=1$ and -1 . The average Nusselt number decreases when increasing the Richardson number in the forced convection region. There is no considerable variation in the total heat transfer rate by increasing the Hartmann number from 0 to 50 in the mixed and natural convection regions. The average Nusselt number is almost constant for all values of the Richardson number in the presence of the strength of magnetic field. It is observed that the heat transfer rate inside the cavity is strongly 


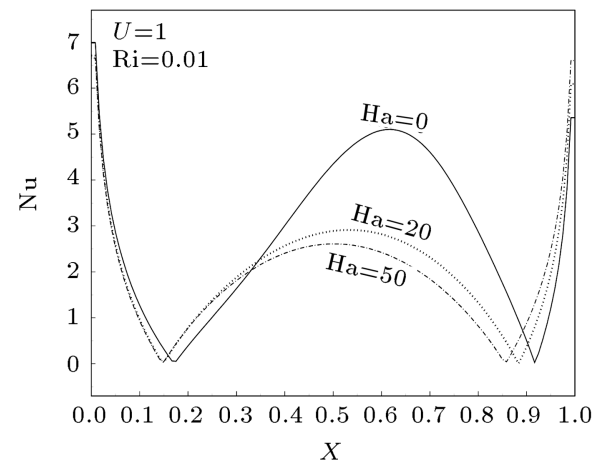

(a)

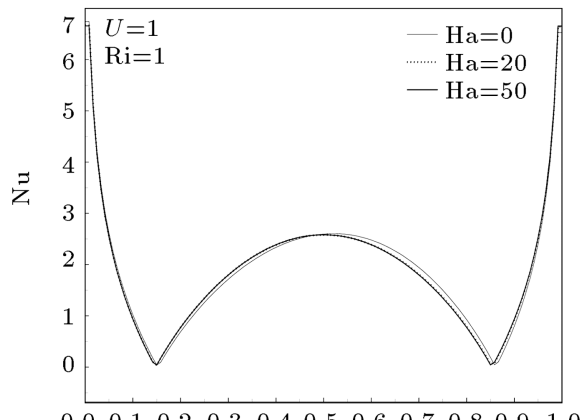

$X$

(c)

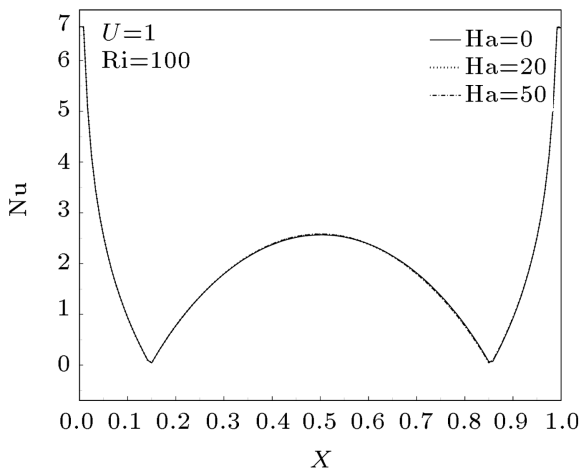

(e)

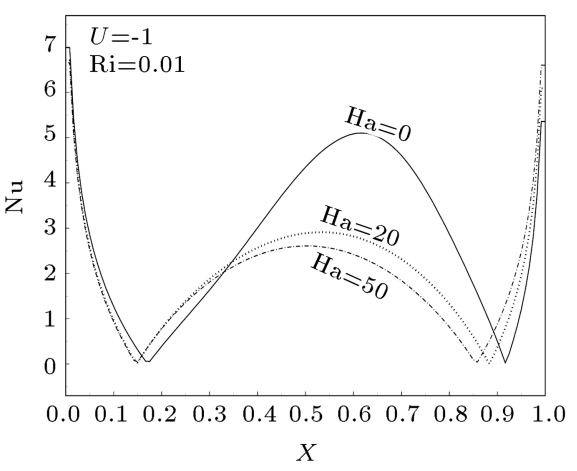

(b)

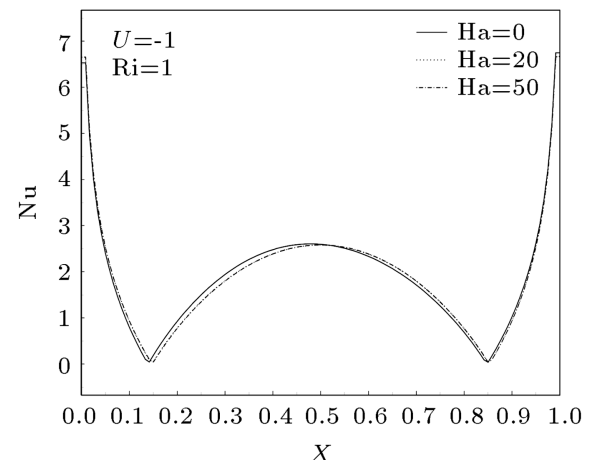

(d)

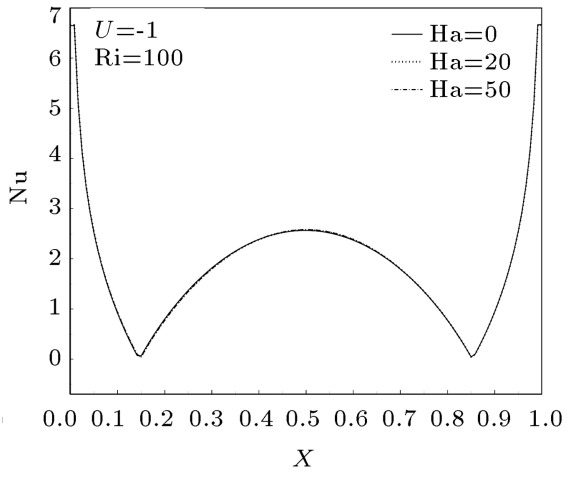

(f)

Figure 6. Local Nusselt number for lid velocities, $U=1$ and $U=-1$.

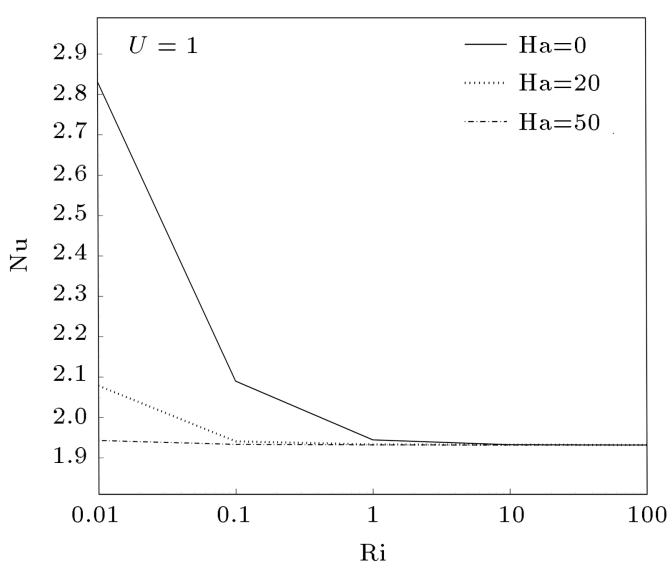

(a)

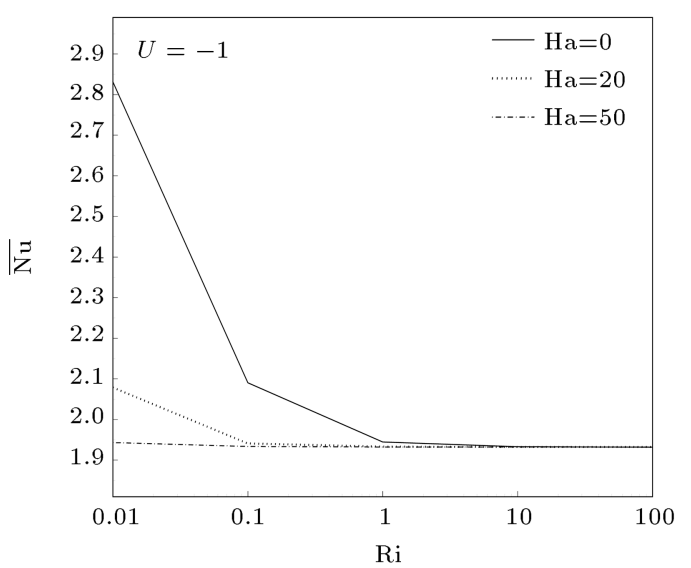

(b)

Figure 7. Average Nusselt number for lid velocities, $U=1$ and $U=-1$. 
affected by the presence of applied magnetic field. It is clear that the average Nusselt number decreases when the Hartmann number and Richardson number are increased.

\section{Conclusions}

This investigation has been concerned with the mathematical modeling of mixed convection heat transfer in a lid-driven cavity with sinusoidal temperature distribution on the bottom wall in the presence of magnetic field.

The following results are obtained from this study.

- The flow behavior is strongly affected inside the cavity by the presence of magnetic field. The mode of convection is not characterized on heat transfer rate in the presence of strong magnetic field;

- The influence of the direction of the moving top wall with velocities $U=1$ and $U=-1$ on heat transfer is not significant. But, the flow field is affected much and flow direction is along the direction of the (top) moving wall;

- The total heat transfer rate is decreased when the Hartmann number and Richardson number are increased.

\section{Nomenclature}

$B_{0} \quad$ Strength of the magnetic field, T

$c_{p} \quad$ Specific heat, $\mathrm{J} /(\mathrm{kg} . \mathrm{K})$

$g \quad$ Gravitational acceleration, $\mathrm{m} / \mathrm{s}^{2}$

Gr Grashof number, $g \beta \Delta T H^{3} / \nu^{2}$

$\mathrm{Ha} \quad H a r t m a n n$ number, $B_{0} L \sqrt{\sigma_{e} / \mu}$

$k \quad$ Thermal conductivity, W/(m.K)

L Cavity length, m

$\mathrm{Nu} \quad$ Local Nusselt number

$\overline{\mathrm{Nu}} \quad$ Average Nusselt number

$p \quad$ Pressure, $\mathrm{Pa}$

Pr Prandtl number, $\nu / \alpha$

Re Reynolds number, $U_{0} L / \nu$

Ri Richardson number, Gr $/ \operatorname{Re}^{2}$

$T$ Dimensionless temperature

$t \quad$ Time, s

$u, v \quad$ Velocity components, $\mathrm{m} / \mathrm{s}$

$U, V \quad$ Dimensionless velocities

$U_{0} \quad$ Lid velocity, $\mathrm{m} / \mathrm{s}$

$x, y \quad$ Cartesian coordinates, $\mathrm{m}$

$X, Y \quad$ Dimensionless coordinates, $(x, y) / L$

\section{Greek symbols}

$\beta \quad$ Coefficient of thermal expansion, $\mathrm{K}^{-1}$

$\zeta \quad$ Dimensionless vorticity

$\theta \quad$ Temperature, $\mathrm{K}$

$\mu \quad$ Dynamic viscosity, $\mathrm{Pa} \mathrm{s}$

$\nu \quad$ Kinematic viscosity, $\mathrm{m}^{2} / \mathrm{s}$

$\rho \quad$ Density, $\mathrm{kg} / \mathrm{m}^{3}$

$\sigma_{e} \quad$ Electrical conductivity, $\mathrm{S} / \mathrm{m}$

$\tau \quad$ Dimensionless time, $t U_{0} / L$

$\psi \quad$ Stream function, $\mathrm{m}^{2} / \mathrm{s}$

$\Psi \quad$ Dimensionless stream function, $\psi / L U_{0}$

$\omega \quad$ Vorticity, $1 / \mathrm{s}$

\section{Subscripts}

$\begin{array}{ll}H & \text { Hot wall } \\ C & \text { Cold wall } \\ 0 & \text { Reference state }\end{array}$

\section{References}

1. Prasad, A.K. and Koseff, J.R. "Combined forced and natural convection heat transfer in a deep lid-driven cavity flow", Int. J. Heat Fluid Flow, 17, pp. 460-467 (1996).

2. Khanafer, K.M., Al-Amiri, A.M. and Pop, I. "Numerical simulation of unsteady mixed convection in a driven cavity using an externally excited sliding lid", Euro. J. Mech. B/Fluids, 26, pp. 669-687 (2007).

3. Waheed, M.A. "Mixed convective heat transfer in rectangular enclosures driven by a continuously moving horizontal plate", Int. J. Heat Mass Transfer, 52, pp. 5055-5063 (2009).

4. Sivakumar, V., Sivasankaran, S., Prakash, P. and Lee, J. "Effect of heating location and size on mixed convection in lid-driven cavities", Comput. Math. Appl., 59, pp. 3053-3065 (2010).

5. Abedini, A., Rahimi, A.B. and Kianifar, A. "Numerical study of mixed convection in the annulus between eccentric rotating cylinders", Scientia Iranica $B$, 21(4), pp. 1403-1414 (2014).

6. Rudraiah, N., Barron, R.M., Venkatachalappa, M. and Subbaraya, C.K. "Effect of a magnetic field on free convection in a rectangular enclosure", Int. J. Eng. Sci., 33, pp. 1075-1084 (1995).

7. Al-Na jem, N.M., Khanafer, K.M. and El-Refaee, M.M. "Numerical study of laminar natural convection in tilted enclosure with transverse magnetic field", Int. J. Numer. Methods Heat Fluid Flow, 8, pp. 651-672 (1998).

8. Chamkha, A.J. "Hydromagnetic combined convection flow in a vertical lid-driven cavity with internal heat 
generation or absorption", Numer. Heat Transfer A, 41, pp. 529-546 (2002).

9. Tagawa, T., Ujihara, A. and Ozoe, H. "Numerical computation for Rayleigh-Benard mass convection of water in a magnetic field", Int. J. Heat Mass Transfer, 46, pp. 4097-4104 (2003).

10. Sarris, I.E., Kakarantzas, S.C., Grecos, A.P. and Vlachos, N.S. "MHD natural convection in a laterally and volumetrically heated square cavity", Int. J. Heat Mass Transfer, 48, pp. 3443-3453 (2005).

11. Mittal, V., Baig, M.F. and Kant Khan, B. "Buoyancydriven convection in liquid metals subjected to transverse magnetic fields", J. Indn. Inst. Sci., 85, pp. 119$129(2005)$.

12. Hossain, A.M., Hafiz, M.Z. and Rees, D.A.S. "Buoyancy and thermo capillary driven convection flow of an electrically conducting fluid in an enclosure with heat generation", Int. J. Ther. Sci., 44, pp. 676-684 (2005).

13. Xu, B., Li, B.Q. and Stock, D.E. "An experimental study of thermally induced convection of molten gallium in magnetic fields", Int. J. Heat Mass Transfer, 49, pp. 2009-2019 (2006).

14. Jalil, J.M. and Al-Tae'y, K.A. "The effect of nonuniform magnetic field on natural convection in an enclosure", Numer. Heat Transfer A, 51, pp. 899-917 (2007).

15. Sivasankaran, S. and Ho, C.J. "Effect of temperature dependent properties on MHD convection of water near its density maximum in a square cavity", Int. J. Ther. Sci., 47, pp. 1184-1194 (2008).

16. Sivasankaran, S., Bhuvaneswari, M., Kim, Y.J., Ho, C.J. and Pan, K.L. "Numerical study on magnetoconvection of cold water in an open cavity with variable fluid properties", Int. J. Heat Fluid Flow, 32, pp. 932942 (2011).

17. Sivasankaran, S. and Bhuvaneswari, M. "Effect of thermally active zones and direction of magnetic field on hydromagnetic convection in an enclosure", Ther. Sci., 15, pp. 367-382 (2011).

18. Sankar, M., Venkatachalappa, M. and Do, Y. "Effect of magnetic field on the buoyancy and thermo capillary driven convection of an electrically conducting fluid in an annular enclosure", Int. J. Heat Fluid Flow, 32, pp. 402-412 (2011).

19. Kefayati, Gh.R. "Lattice Boltzmann simulation of natural convection in a nanofluid filled inclined square cavity at presence of magnetic field", Scientia Iranica, $B$, 20(5), pp. 1517-1527 (2013).

20. Sheikholeslami, M. and Ganji, D.D. "Magneto hydrodynamic flow in a permeable channel filled with nanofluid", Scientia Iranica, B, 21(1), pp. 203-212 (2014).

21. Deng, Q.H. and Chang, J.J. "Natural convection in a rectangular enclosure with sinusoidal temperature dis- tributions on both side walls", Numer. Heat Transfer A, 54, pp. 507-524 (2008).

22. Sivasankaran, S., Sivakumar, V. and Prakash, P. "Numerical study on mixed convection in a lid-driven cavity with non-uniform heating on both side walls", Int. J. Heat Mass Transfer, 53, pp. 4304-4315 (2010).

23. Bhuvaneswari, M., Sivasankaran, S. and Kim, Y.J. "Magneto-convection in a square enclosure with sinusoidal temperature distribution on both side walls", Numer. Heat Transfer A, 59, pp. 169-184 (2011).

24. Sivasankaran, S., Malleswaran, A., Lee, J. and Sundar, P. "Hydro-magnetic combined convection in a liddriven cavity with sinusoidal boundary conditions on both sidewalls", Int. J. Heat Mass Transfer, 54, pp. $512-525$ (2011).

25. Sivasankaran, S. and Pan, K.L. "Numerical simulation on mixed convection in a porous lid-driven cavity with non-uniform heating on both side walls", Numer. Heat Transfer A, 61, pp. 101-121 (2012).

26. Sivakumar, V. and Sivasankaran, S. "Mixed convection in an inclined lid-driven cavity with non-uniform heating on both side walls", J. Appl. Mech. Tech. Phys., 55(4), pp. 634-639 (2014).

27. Sivasankaran, S. and Pan, K.L. "Natural convection of nanofluids in a cavity with non-uniform temperature distributions on side walls", Numer. Heat Transfer A, 65, pp. 247-268 (2014).

\section{Biographies}

Sivanandam Sivasankaran received his MSc, MPhil, and $\mathrm{PhD}$ degrees from Bharathiar University, India, in 2000, 2002, 2006, respectively. After that, he received Post-Doctoral Fellowship from National Cheng Kung University, Taiwan, and National Taiwan University, Taiwan. He was a Research Professor at Yonsei University, South Korea. He was an Assistant Professor at Sungkyunkwan University, South Korea. Presently, he is a Senior Lecturer in the Institute of Mathematical Sciences, University of Malaya, Malaysia. He is a member of editorial board in several international journals and reviewer of more than 20 international journals. His areas of interest are convective heat and mass transfer, CFD, nanofluids, micro-channel heat sinks, and Porous Media.

Seenapuram Shanmugam Ananthan received his MSc degree in Mathematics from Bharathiar University, India, in 2000 and his MPhil degree in Mathematics from Alagappa University, India in 2003. His research interest is convective flow and heat transfer in enclosures.

Abdul Kaffoor Abdul Hakeem received his MSc 
degree in Mathematics from Bharathiar University, India, in 2002, his MPhil degree in Mathematics from Bharathiar University, in 2004, and his PhD degree in Fluid Dynamics from Bharathiar University, in 2008. $\mathrm{He}$ is serving the Department of Mathematics, Sri
Ramakrishna Mission Vidyalaya College of Arts and Science, Coimbatore, India Affiliated to Bharathiar University, as an Assistant Professor since 2008. His research interests are convection in cavity and boundary layer flow. 